\title{
Conduction of Electric Current to Organic Molecules observed by Carrier Transport Mobility Measurements
}

\author{
Toshiki Komatsu* \\ Corporate Research and Development \\ JNC Corporation \\ 290-8551 Ichihara Chiba, Japan
}

* To whom correspondence should be addressed:

Toshiki Komatsu (komatsu@jnc-corp.co.jp) 


\section{Abstract}

Organic semiconductors have been studied for many years, but electric current conduction through organic materials has proven difficult in practice. To evaluate the carrier mobility of newly synthesized modified polyacenes, we prepared suitable techniques for determining the physical properties of the polyacenes from electrical measurements. In a transparent electrode cell based on a low-melting-point material, we observed the dark-current dependence on the cooling rate and evaluated the sample impurity. We then developed a vertical field-effect transistor (FET) device for highmelting-point materials that easily measures the transconductance of a single drop of a sample solution. The vertical FET device provides indirect evidence of Marcus' theory of electric conduction phenomena in aggregated organic materials. 


\section{Introduction}

The semiconducting properties of organic matter have attracted much interest in modern organic chemistry ${ }^{1}$. The ultimate goal is to synthesize $\Pi$ electron-system solids ${ }^{2}$. Like devices formed from inks and plastics, devices based on organic semiconductors are expected to be prepared by fast and cheap printing processes ${ }^{3}$.

Almost all aromatic molecules are semiconductive, including benzene iodide, the smallest aromatic ${ }^{4}$. Currently, the semi-conductivities of organic molecules are judged by their carrier mobility. The measurement methods are summarized below:

1. Under excitation by a light or electron beam, carrier mobility can be detected as a change in the microwave absorption ${ }^{5}$ or apparent electrical conductivity of a solid material ${ }^{6,7}$.

2. The electric characteristics can be measured in electronic devices constructed from the material ${ }^{8}$.

However, when the experimental results of solid excitation and electronic devices deviate from the expected results, the values of the inherent physical properties cannot be fully trusted, and truer values should be sought.

Our group has been acquiring organic semiconductor molecules suitable for printing by collaborative research institutes. Such molecules possess an aromatic semiconductive core with highly soluble side chains, which lower the melting point and facilitate the production of ink.

First, we attempted to purify large amounts of a tetracene compound with low melting point and high solubility, and performed mobility measurements under a solidexcitation photocurrent. The secondarily obtained pentacene was present in very small amounts. Despite its high solubility, the tetracene compound has a melting point above its decomposition point. A new field effect transistor (FET) device was formed from non-heated tetracene solution and its mobility was estimated from its electrical characteristics.

This device challenges the conventional idea that organic compounds cannot conduct electricity. Although our experiments provide no direct evidence of electrical conductivity, a feasible hypothesis admits current to the devices. Here we describe the hypothesis and a model of semiconductivity in an organic compound that unifies the interpretations of both solid excitation experiments and electronic device characterizations.

\section{Experimental}

Materials

1,4,7,10-Tetrahexyltetracene [Compound (1)] was provided by Professor 
Kitamura of Hyogo Prefectural University9. This product was purified twice in a column chromatograph and recrystallized once after retrieval. In the solid-excitation experiment, Compound (1) was measured as received. For a more precise analysis, Compound (1) was recrystallized four times in 2-propanol.

2,3,9,10-Tetramethoxycarbonylpentacene [Compound (2)] was provided by Professor Takaguchi of Okayama University ${ }^{10}$ and was used as received.

4-Octyloxy-4'-(mercaptomethyl) biphenyl [Compound (3)] was synthesized from 4-Octyloxy-4'-(bromomethyl) biphenyl following Hart et al ${ }^{11}$. Compound (3) was purified once by column chromatography and then recrystallized.

\section{Mass spectrometry}

Time-of-flight secondary ion mass spectrometry (ToF-SIMS) measurements were performed by IONTOF TOF-SIMS ${ }^{3}$ using a $\mathrm{Gd}^{+}$ionization source for organic species. Metallic element was analyzed with a SIMS tool (Cameca Instruments IMS-7F). Both were provided by Hitachi Urban Support Ltd. (Chiba, Japan) and Japan Display Inc. (Chiba, Japan), respectively.

Photocurrent observation under solid-surface excitation

The photocurrent was observed in a glass test cell with a $10-\mu \mathrm{m}$ gap between two indium-tin-oxide (ITO) mortal electrodes inside. Compound (1) was melted at $100^{\circ} \mathrm{C}$ in a hot-stage microscopy (Mettler Toledo FP-81) and absorbed into the cell under capillary action. The sealed cell was heated to the isotropic phase and the grain size of the crystal was confirmed after rapidly cooling on the table. The sample was gradually cooled at $10^{\circ} \mathrm{C} / \mathrm{min}$ in the $\mathrm{FP}-81$. The TOF transient photocurrent was recorded under a Laser NdIII-YAG (Spectra Physics GCR-2 $532 \mathrm{~nm}$ ) and a Transient Storage Scope (500 MHz; Teledyne LeCroy Inc.) in the Arai Laboratory at the University of Tsukuba, Japan ${ }^{12}$. The applied DC voltage was $30 \mathrm{~V}$.

Preparation of electronic devices and observation of their electrical properties

The electronic device was a three-wire-crossed type vertical FET, prepared as follows.

\section{(I) Preparation of the surface-treated metal electrode}

The metal electrodes were formed from pure gold wire (AU-171481; Nilaco Corporation, Tokyo, Japan) of diameter $1.0 \mathrm{~mm}$ and length $100 \mathrm{~mm}$. Each metal electrode was cut to a length of $20 \mathrm{~mm}$ and fold in half. A self-assembled monolayer was formed on the gold-wire surface by immersing its bent portion in $3 \mathrm{~mL}$ of an ethanol solution containing $10 \mathrm{mg}$ of dissolved Compound (3). After immersion overnight, the gold wire was removed from the ethanol solution, washed in ethanol, and dried. The sections above the surface-treated section were used as the drain and source electrodes.

A gold wire was soldered to a copper micro grid for electron microscopy 
observations (\# 09-1013 100 mesh made by Oken Shoji Co., Ltd., Tokyo, Japan) and an insulating layer was formed by applying Sunhayato VA-30 coating agent to the grid part followed by air drying. The coating process was performed twice.

\section{(ii) Fabrication of the Field Effect Transistor (Vertical FET)}

A drain electrode, a gate electrode and a source electrode were sequentially stacked on a clean slide glass and the end portion was fixed with a dual-liquid-type epoxy adhesive (Araldite; see Figure 1). The FET was placed in a fixture for testing (16442B, Agilent Technologies Japan, Ltd.) and wired to Semiconductor Device Parameter Analyzer (B1500, Agilent Technologies Japan, Ltd.). Next, $0.1 \mathrm{~g}$ of a solution prepared by dissolving ca. $0.1 \mathrm{mg}$ of Compound (1) in $1 \mathrm{~mL}$ of methyl ethyl ketone (manufactured for electronic materials by Wako Pure Chemical Industries) was dropped between the source and drain electrodes, forming a vertical FET containing a150- $\mu$ m-thick film of the organic semiconductor. A similar vertical FET was obtained by dropping $250 \mu \mathrm{g}$ of Compound (2) dissolved in ca. $0.1 \mathrm{~mL}$ of methyl ethyl ketone between the electrodes. Again, the source and drain electrodes were separated by 150 $\mu \mathrm{m}$.
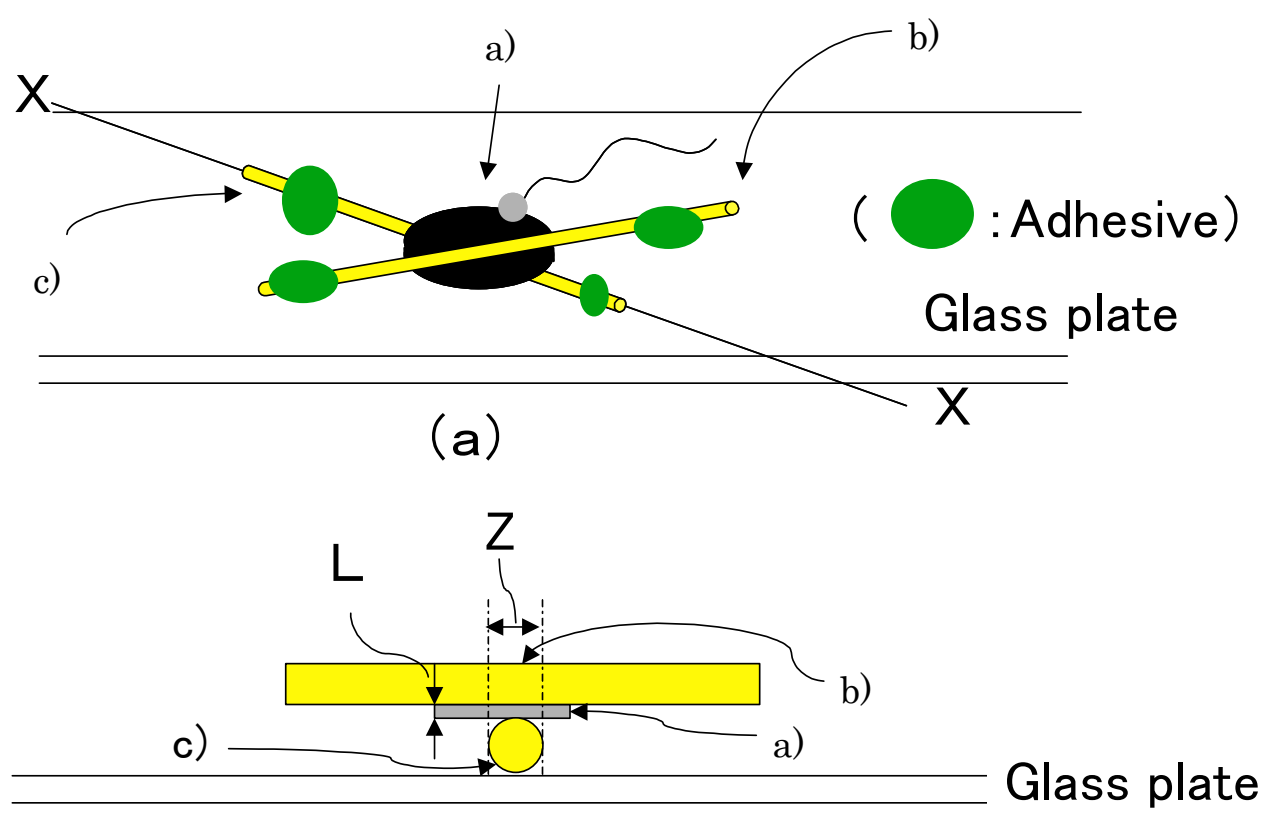

(b)

Figure 1. Schematic of the vertical FET (a) and its cross section along the $\mathrm{X}$-axis (b). Labels: a) Gate electrode: copper TEM grid insulated by radio varnish, cable soldered; b) and c) Source and drain electrode, respectively, formed of gold wire covered by a thin gold-thiol-bonded layer of Compound (3). At a wire radius of $Z$ and gate 
thickness of $L$, the carrier mobility is constant.

\section{(iii) Evaluation of Drain Current of the Vertical FETs}

The semiconductor characteristics of the vertical FETs were evaluated in a dark ambient atmosphere. The vertical FET obtained in subsection (ii) was wired to a B 1500 three-terminal semiconductor device analyzer (Agilent Technologies Japan, Ltd. ). The organic semiconducting thin film was formed by dropwise addition of $0.1 \mathrm{~g}$ of Compound (2) dissolved in methyl ethyl ketone, and the voltage sweep was immediately applied by a B1500 three-terminal semiconductor device analyzer (Agilent Technologies, Inc.). The drain current (pinch-off current) was measured at different gate voltages by the device analyzer. In these measurements, the source electrode was set to the same voltage as the gate electrode. The square root of the pinch-off current was plotted as a function of gate voltage, and the carrier mobility was calculated from the slope of this graph.

\section{Results}

Relationship between dark current and crystallization speed in the photocurrent observation cell

Figure 2 plots the time course of the TOF current in the sample in which Compound (1) was gradually cooled at $5^{\circ} \mathrm{C} / \mathrm{min}$ in $\mathrm{FP}-81$. From this plot, the mobility was determined as $0.038 \mathrm{~cm}^{2} / \mathrm{sec}$. Meanwhile, the quenched samples exhibited a dark current and dielectric breakdown occurred under light irradiation (Figure 3). 


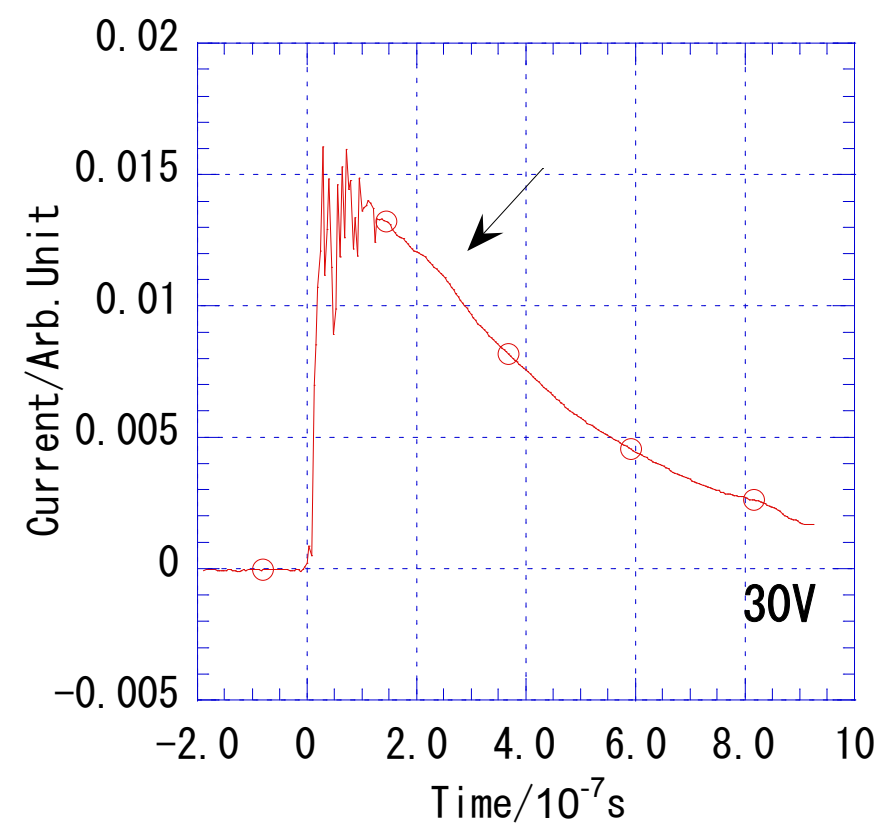

a)

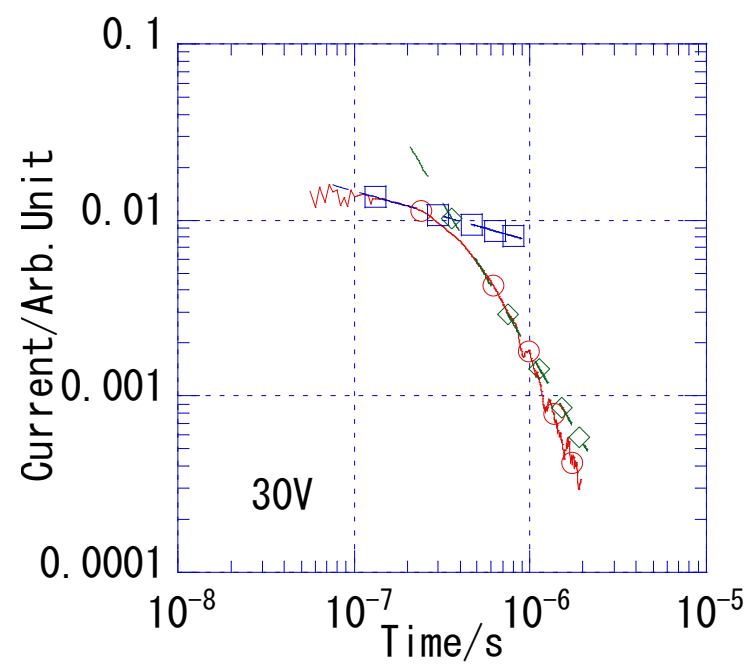

b)

Figure 2. Carrier mobility measurements of Compound (1). The flexion point is unclear in the linear plot (a), but appears in the log-log plot (b). 


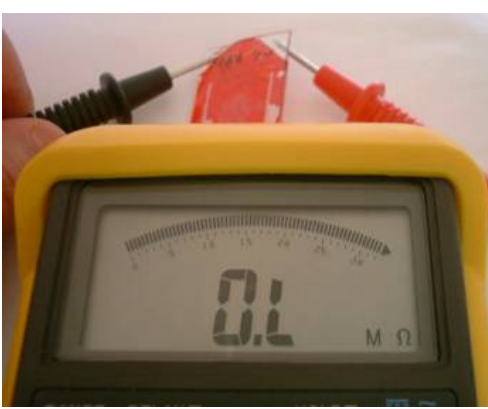

a)

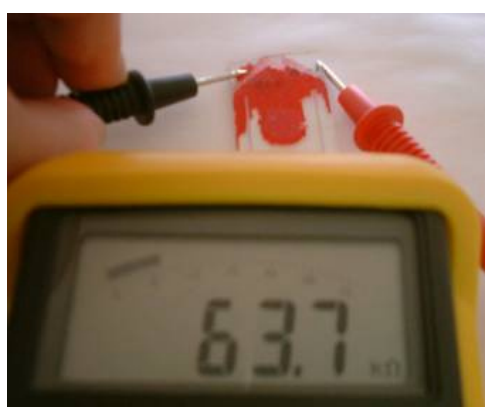

b)

Figure 3. Compound (1) sealed in the TOF cell and (a) slowly cooled by $5{ }^{\circ} \mathrm{C} / \mathrm{min}$ in FP-81; (b) quenched on the ambient desk. The cooling rate affects the distribution of conductive impurities.

\section{Component analysis of crystals and mother liquor after four recrystallizations}

Compound (1) and the components of the mother liquor were analyzed by TOF-SIMS and SIMS. The major impurities were siloxanes, alkanes, and alkaline metals.

\section{Cation analysis}

Monovalent cation dimers in the crystals were detected at full scale under the relevant ionization conditions (see Figure 4). Siloxane and Compound (1) were detected in the mother liquor, but no multimer crystals were detected. 

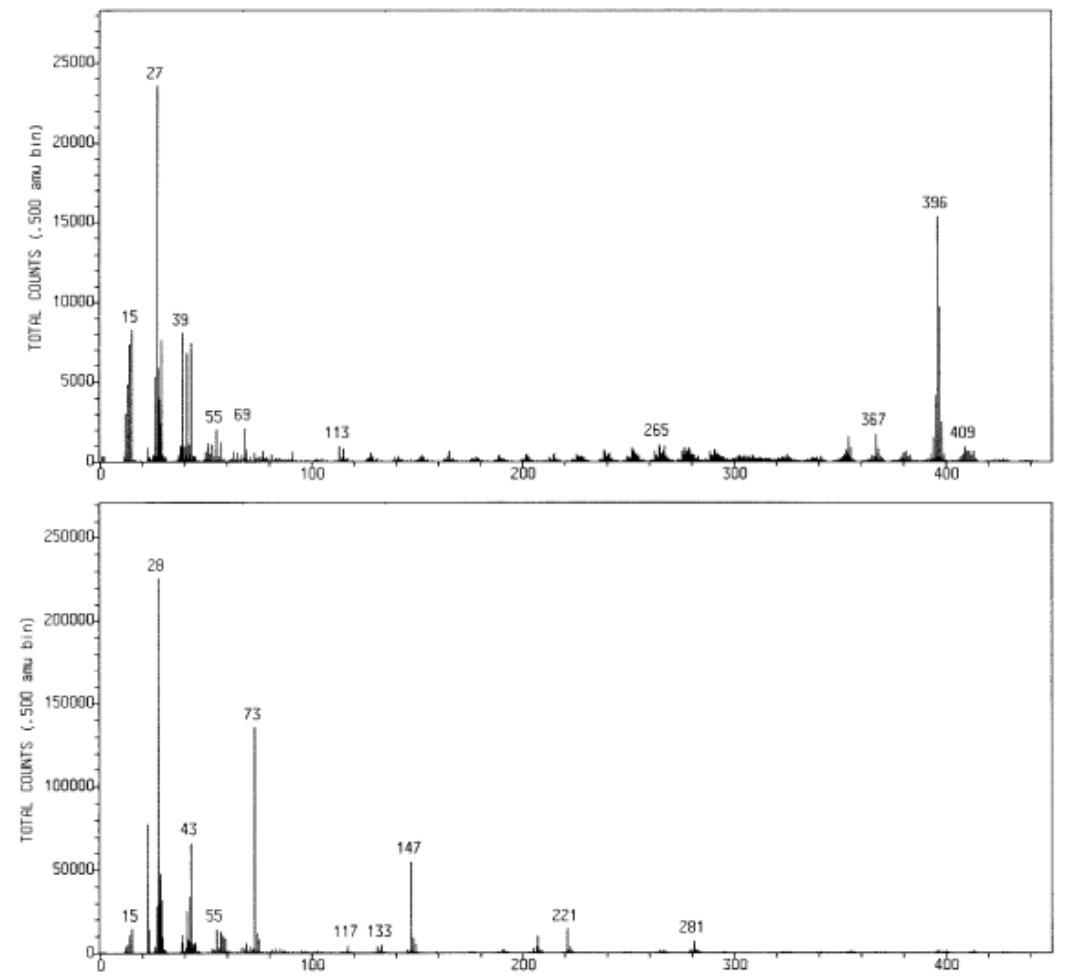

a)

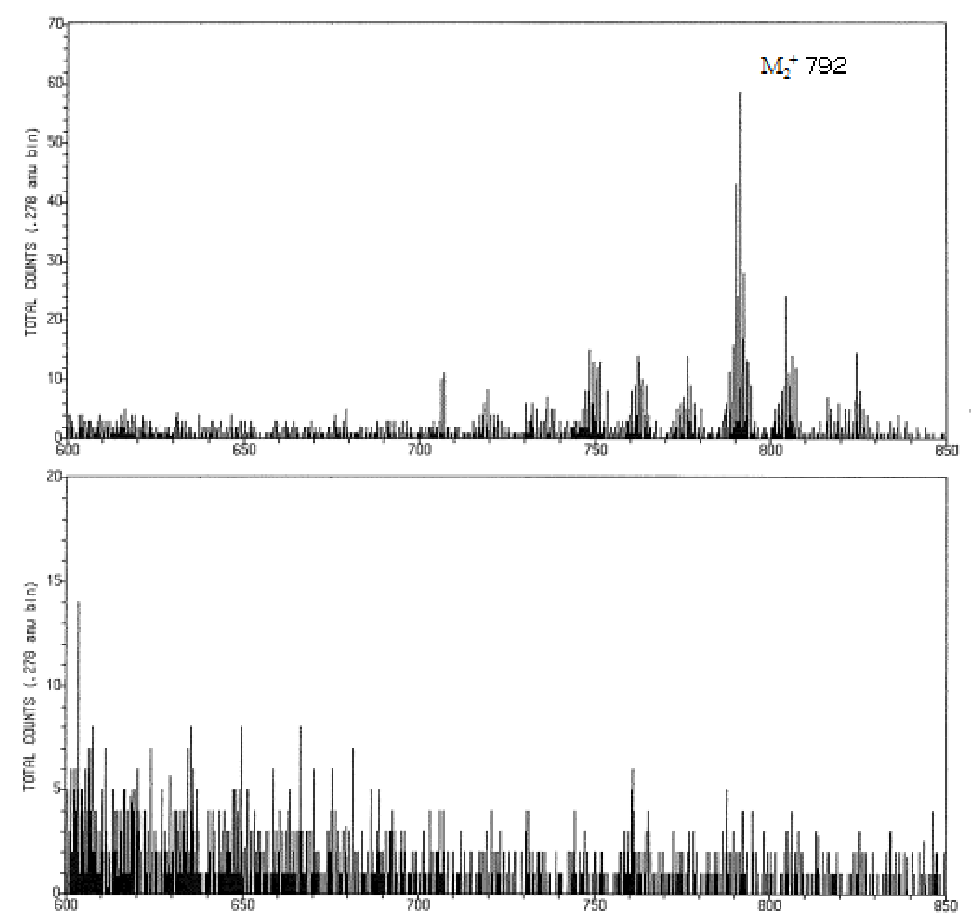

b)

Figure 4. Cation TOF-SIMS spectra of crystals (upper) and mother liquor (lower) of Compound (1). In (a), siloxane impurities are found. In (b), dimer monocation 
is observed.

\section{Anion analysis}

No cation multimers were detected in the crystals, and a strong $\mathrm{CH}$ peak appeared in the spectrum of the mother liquor (see Figure 5).
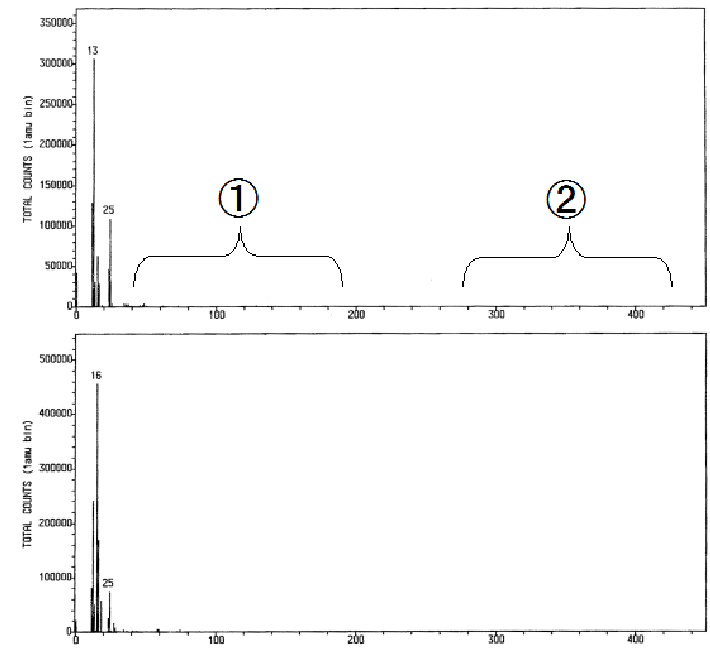

a)
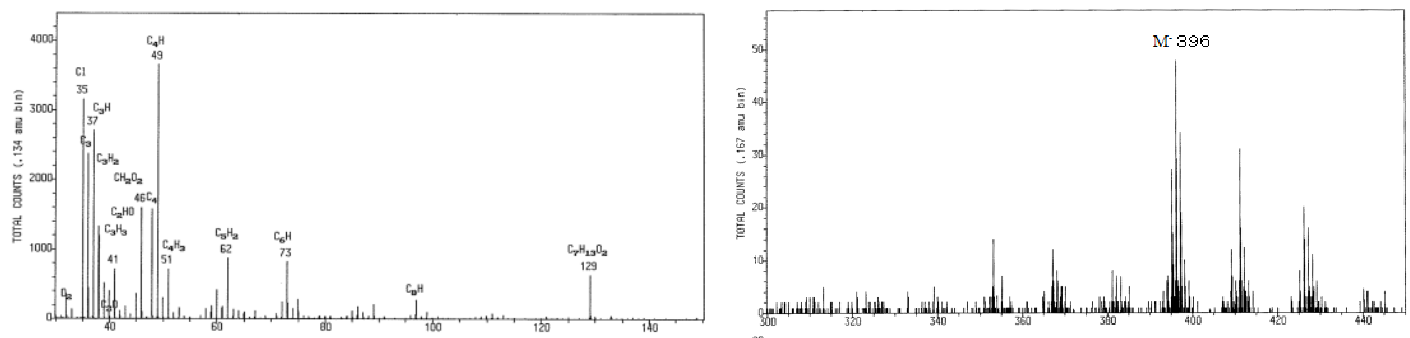

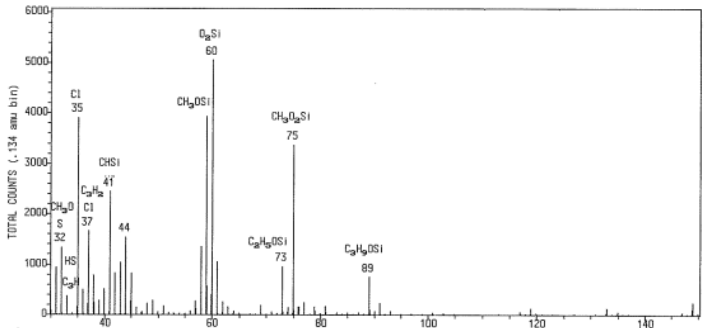

b)

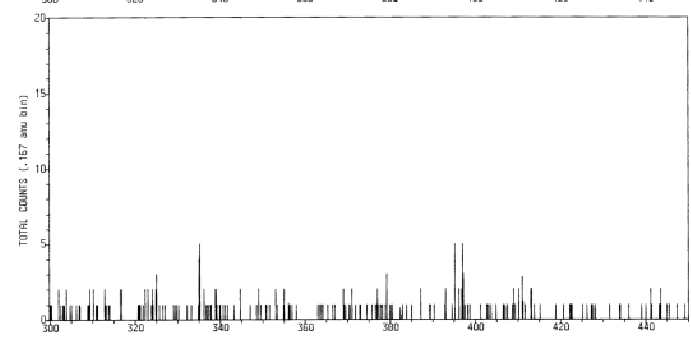

c)

Figure 5. Anion TOF-SIMS spectra of crystals (upper) and mother liquor (lower) of Compound (1). In (a), a strong $\mathrm{CH}$ peak is observed. In (b), siloxanes impurities are observed. In (c), the M-peak characterizes crystals. 


\section{SIMS analysis}

Lithium, sodium, potassium, and zinc appeared in the SIMS spectrum after recrystallization (Figure 6)

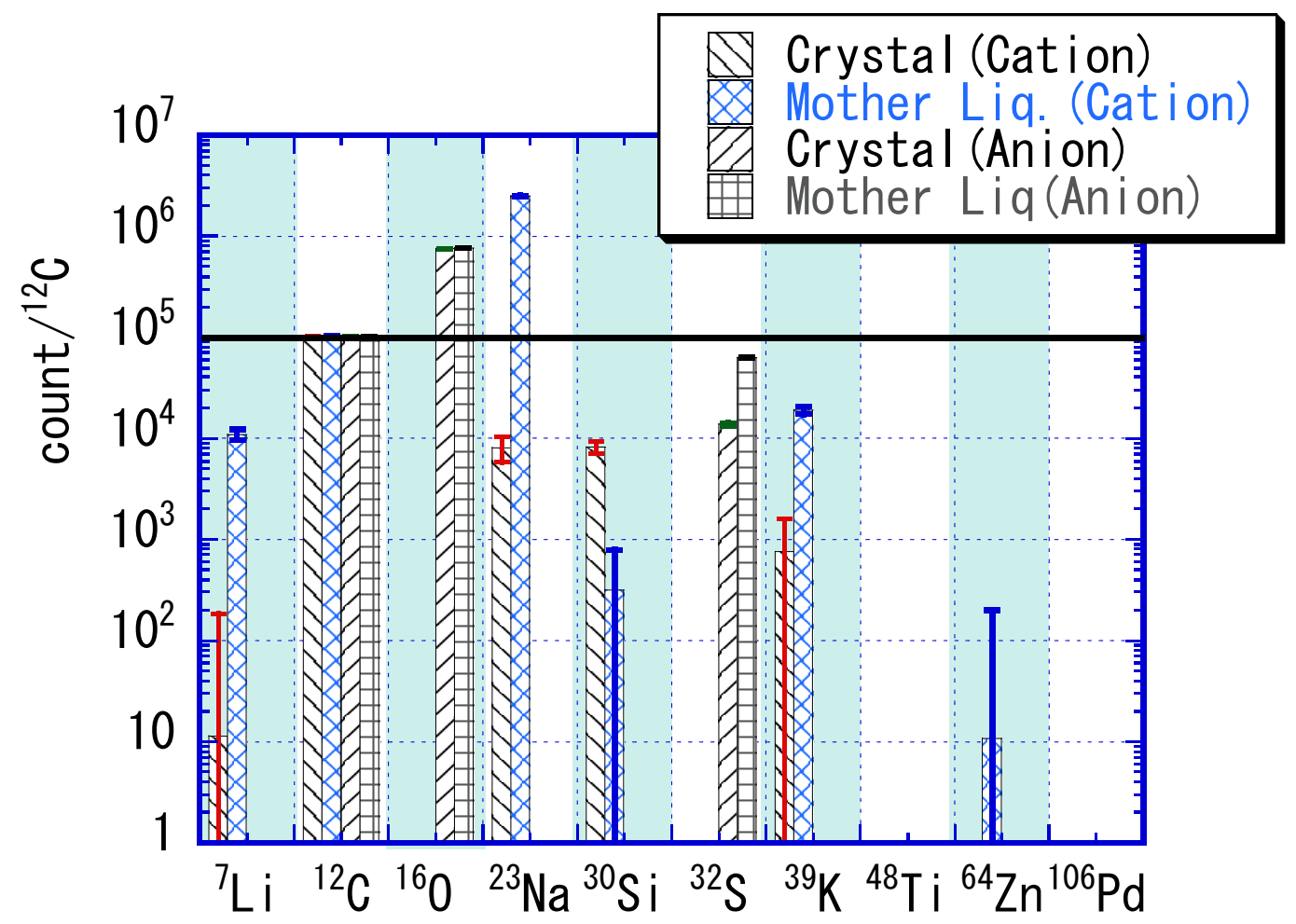

Figure 6. SIMS ion counts of Compound (1) normalized by $12 \mathrm{C}$

\section{Current-voltage characteristic of electronic device and its cycle change}

A solution of Compound (2) was dropped onto the vertical FET as shown in Figure 1. A gold wire surface-modified with Compound (3) was connected to the gate electrode and the measurement button was operated immediately and repeatedly. The FET response was delayed until the third sweep (see Figure 7) and was absent in the fifth sweep. Other FETs showed reliable performance and reproducibility (see Figure 8 and 9). 

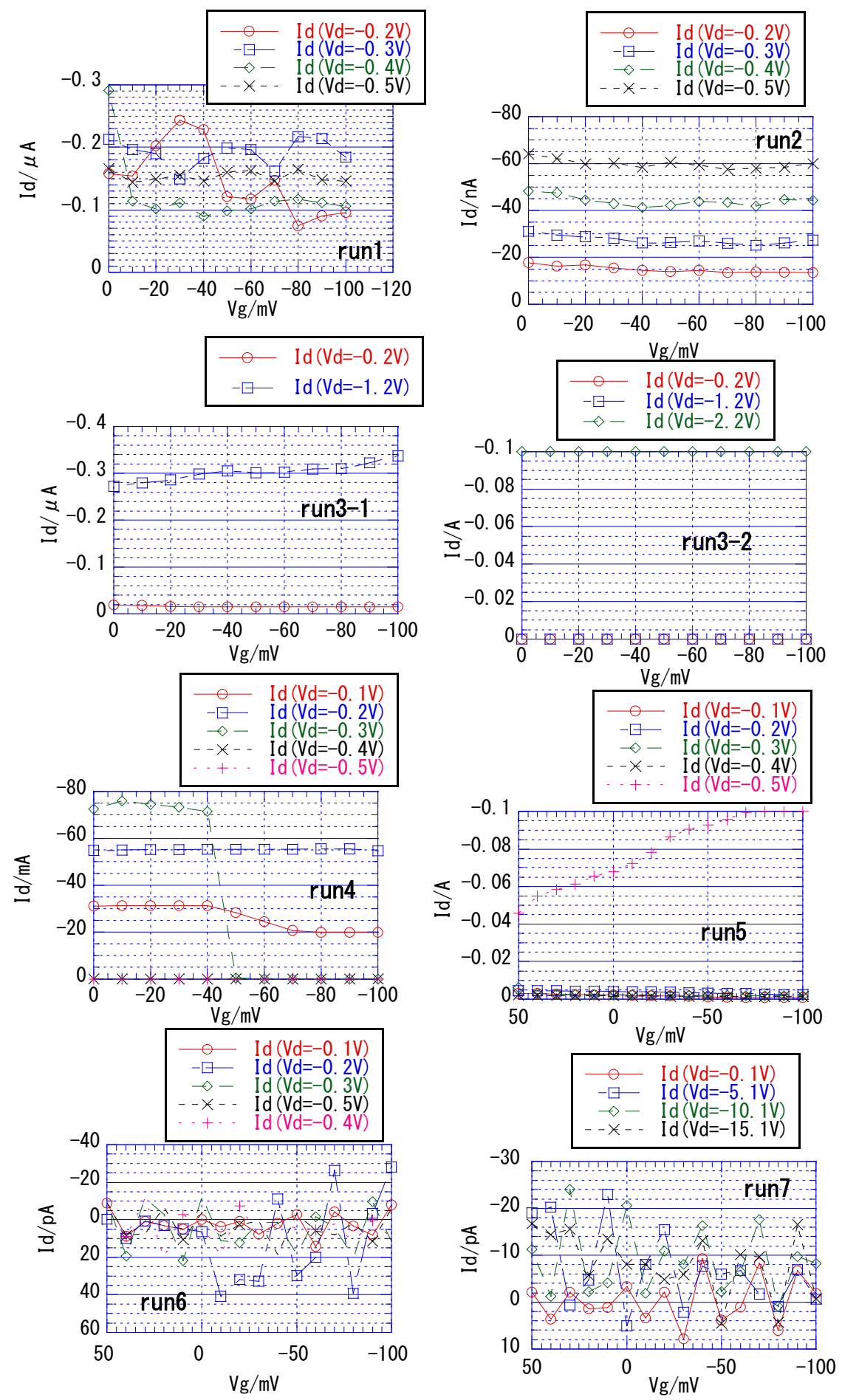

Figure 7. $I_{\mathrm{d}}-V_{\mathrm{g}}$ curves of a vertical FET based on Compound (2) during repeated measurements. In the first and second runs, the diluted solution does not respond. In the third run, wet crystals produce an over-scaled current. On the fourth run, the crystal has dried and appears to become oxygenated. A high voltage elicits a temporary current in the fifth run, before breakdown occurs. 


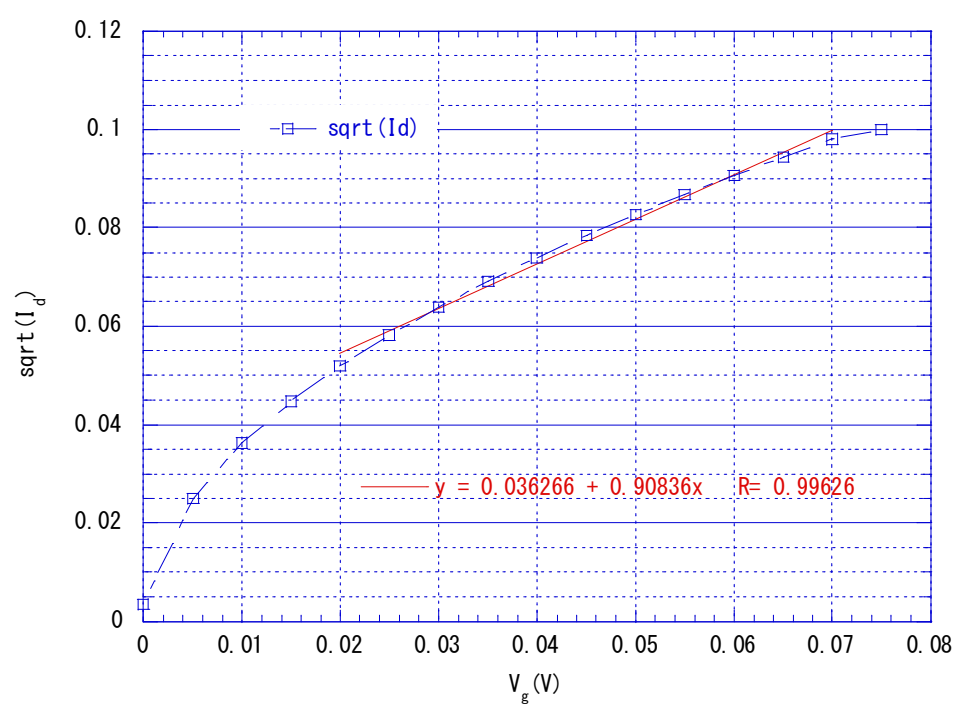

Figure 8. Square root of the pinch-off current versus gate-voltage of the vertical FET based on Compound (2) in the third run

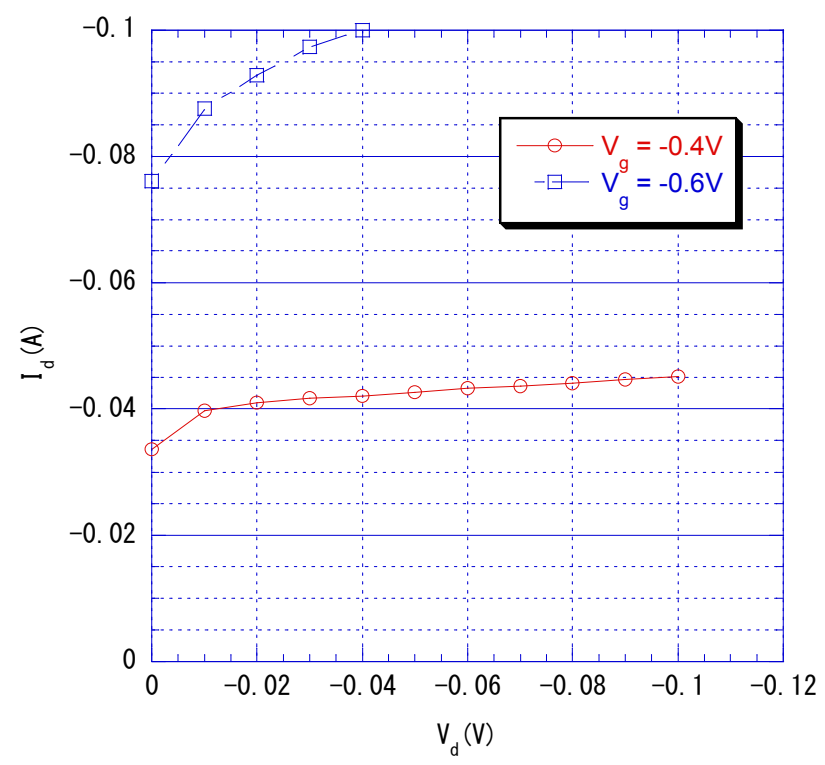

Figure 9 Another $I_{d}-V_{g}$ curve of the vertical FET based on Compound (2), third run. Milli-ampere currents were observed at milli-voltages. 
The organic semiconductor thin film was visually observed after the measurements. Many microcrystals were precipitated and no powder was dislodged when pressed by the finger. Conversely, the raw gold wire repelled the organic solvent, no crystals were adhered, and electrical continuity was not achieved.

After the measurements, crystal precipitates were affirmed by visual observation of the organic semiconductor thin film, but the crystals collapsed under light finger impact.

The gate thickness of the vertical FET was calculated as $L=L_{1}+L_{2}$, where $L_{1}$ is distance between source and gate electrode and thickness of gate electrode and $L_{2}$ denotes distance between gate and drain electrode. The carrier mobility $\mu\left(\mathrm{cm}^{2} / \mathrm{Vs}\right)$ in the vertical FET was then calculated as

$$
\mu=\left(L \times a^{2}\right) /\left(Z \times \mathrm{C}_{0}\right)
$$

where $Z$ is the width of the source/drain electrodes $(1.0 \mathrm{~mm}) \mathrm{a}$ is the slope of the square root of pinch-off current versus gate voltage, and $C_{0}$ is the capacitance $\left(4.27 \times 10^{-2}\right.$ $\mathrm{F} / \mathrm{cm}^{2}$ ). From the slope of the square root of the pinch-off current versus gate voltage, the carrier mobility of Compound (2) was obtained as $0.021 \mathrm{~cm}^{2} / \mathrm{Vs}$ (see Figure 8)

\section{Discussion}

Starting point

The aim of this research was to evaluate whether carrier mobility is an intrinsic property of an organic FET.

The earliest FETs were prepared only by the vacuum evaporation method and no preparations from solution have been reported. We therefore began our experiment by accumulating TOF data.

\section{Static charge injection from the ITO surface}

TOF data are collected under ideal charge-injection conditions because the charges in the material are directly separated under light, regardless of the state of the electrode.

As Compound (1) has a low melting point and was easily contained in the photoconductive cells, its carrier mobility could be measured by TOF photocurrent. Dielectric breakdown is induced by differences in the physical properties of a material, which depend only on the cooling rate in the same sample. Purification by recrystallization was performed in alcohol to extract any impurities from the compound. Analysis of impurities and discovery of cluster ions of organic semiconductors

In general, the recrystallization method allows quantitative recovery of a material. The impurity component is adequately removed by distilling off the solvent of 
the mother liquor. When crystals of Compound (1) and the mother liquor were analyzed by TOF-SIMS and SIMS, some characteristic behaviors emerged. The conductive impurities appeared to be alkaline metals.

In Compound (1), the multimeric cations allowed fast passage of charge carriers and the impurity removal greatly changed the behavior of the organic semiconductor. This experiment achieved only dimers but if the purity can be further improved, an oligomer and multimer complex can form, generating a large single semiconductive species.

\section{TOF-SIMS Cation Analysis}

The pure crystals appeared as monovalent cation dimers at full scale under the ionization conditions. This observation indicates that the conductivity unit of organic semiconductors can be changed by improving the purity.

The mother liquor may have been contaminated by the silicone grease used in the synthesis or elution from the silica gel used in the preparation. The cause of dielectric breakdown was judged as silicone compounds.

\section{TOF-SIMS Anion Analysis}

No multimers in the crystals were detected in the TOF-SIMS anion analysis. Whether the purity was insufficient or whether only monomer is stable in this compound cannot be discerned at this time.

The mother liquor yielded a strong peak derived from $\mathrm{CH}$, which was likely contributed by alkanes.

\section{SIMS analysis}

Recrystallization effectively extracted all impurities except silicon and oxygen. Especially, it removed alkaline metals from the crystal. The dark current in the quenched samples (Figure 3) apparently arose from distribution of alkaline metals in the gap space, which depended on the cooling rate.

\section{Conductivity model of the modified organic semiconductor}

Compound (2), with a melting point of $300^{\circ} \mathrm{C}$ or higher, could not be confined in the photoconductive cell.

Furthermore, we needed to develop a FET measurement device for solutions because the product weight was far below the lower limit required for vapor deposition.

The TOF photocurrent can be measured even when the electrodes are insulated, because the light beam separates the charges and transient conduction occurs.

Organic substances are generally considered as insulators. When the lead of a tester is applied to the powder of an organic compound, no conductivity is observed. However, the conductivity of tetracene or pentacene in a reagent bottle has not been tested in this way, although vapor-deposited pentacene is known as an organic 
"semiconductor". Clearly, there exists a discrepancy between the theory and the device fabrication.

To resolve this discrepancy, we invoke Marcus' theory in bulk. It is assumed that band-gap theory is inapplicable to molecular crystals with interatomic distances of $3 \AA ̊$ or more. In the ground state, molecular crystals behave as insulators. Conversely, in Marcus' theory, the cation-anion species generated by one-electron oxidation reduction can couple the radical electrons in the highest occupied molecular orbital/lowest unoccupied molecular orbital up to $6 \AA$ atomic distances ${ }^{13,14}$. We considered that intermolecular interactions leading to continuous electron exchange are possible in molecular crystals if a charge-separated state can be continuously formed at the electrode interface ${ }^{15}$.

\section{Charge injection problem of interface in electronic device}

To prove the above hypothesis, we discuss the existence and destruction of the vacuum level on the electrode surface. Considering the reagent bottle with a tester lead and the TOF spectrum, we inferred that the development of a solution FET device is prohibited by the formation of an vacuum interface between the organic material and gold electrode. A pure gold electrode forms a stable chemical bond with an organic thiol compound ${ }^{16}$.

Marcus theory suggests that an FET response can be elicited if an electronic interaction establishes between organic thiol and an organic semiconductor molecule. We therefore checked for FET-like signal responses when a solution of Compound (2) was dropped on the top of the vertical FET.

\section{Implementation of simple and effective electronic device}

Before constructing a vertical FET, we prepared an electrode-deposited gateinsulated silicon substrate as a bottom gate-type FET, and dropped a solution of Compound (2) onto it. The solution spread across the substrate surface and only microcrystals were formed. As no source-to-drain conduction was obtained, the FET response was not observed.

In the planar TFT structure, grasping of the solution probably exhausted the sample before resolution. We therefore abandoned this configuration and instead developed a vertical-type FET. In this configuration, the solution was grasped at the intersection of the conductors and crystallized there.

\section{Behavior and interpretation of electronic devices in air}

As shown in Figure 7, no conduction was observed during the first sweep and the FET response was delayed until the third and fourth sweeps. It was thought that the FET performance emerged through concentration and crystallization of the organic semiconductor thin film. In the initial stage, the organic semiconductor molecules were 
not gathered within $6 \AA ̊$ in the dilute solution, so could not interact.

From the fifth sweep onward, the solvent dried and the organic semiconductor was exposed to the atmosphere. At this time, the organic semiconductor was destroyed by the influence of oxygen and water through the cation species.

After the measurements, an organic semiconductor thin film was visually observed. Further analysis revealed a large number of microcrystals on the film. When impacted with a finger, no powder or similar material was dislodged, confirming the stability of the organic semiconductor thin film.

Later, we found that the surfaces of gold atoms behaved as semiconductors when forming gold-thiol bonds ${ }^{17}$.

However, in the vertical-type FET, the gold wire corresponding to the source/drain electrode was not modified by Compound (3), and the expected FET response was not observed. Furthermore, after the measurements, no crystal precipitation was observed by visual observation of the organic semiconductor thin film. The crystals collapsed under slight finger impact, and mechanical contact with the electrode was not made.

Interpretation of charge mobility

Under the above hypothesis, the activation energy associated with the electron transfer reaction occurs in the portion corresponding to the band gap. Considering the electron transfer reaction in solution, the activation energy was approximated as the thermal energy at room temperature ${ }^{18}$, which is sufficient to establish a semiconductor.

When calculating the mobility of Compound (2), we cannot measure the external dimensions and capacitance between the electrodes of the vertical FET. We therefore assumed the lowest possible values for calculating the mobility (see Figure 1):

- Source and drain electrode length = gold wire thickness

- Inter-electrode gap between source and drain = thickness of gate electrode

- Capacitance $=$ theoretical capacitance when an air capacitor is inserted between the source and drain

The minimum value obtained is stated in the patent ${ }^{10}$.

To optimize this value, we should ideally obtain a relative amount of pure sample. In the simply configured vertical FET with a mA current flow, the mobility was of the order of $10^{-2}$ to $10 \mathrm{~cm}^{2} / \mathrm{Vs}$. The mobility was measurable in completed devices with four samples of microcrystals (total amount $=250 \mu \mathrm{g} !$ ).

\section{Conclusion}

Although we could only indirectly prove the validity of Marcus theory in charge transport phenomena, we assumed some supporting evidences and solved the problem 
of carrier injection. We also developed a measurable organic FET electrode device with one drop of solution between the electrodes.

The mobility values determined by the newly developed method should be compared with those of the conventional vapor deposition method and the TOF photocurrent method.

According to Marcus' theory, current in bulk organic materials can be generated by the following methods:

$>$ Weak ionization between organic bulk and the electrode interface

$>$ Injection of carriers under an electric potential gradient

$>$ Induction of the serial ionization exchange reaction

$>$ Driving of charges to the counter electrode

The TOF photocurrent method is ideal because it does not depend on the charge injection efficiency at the electrode surface. Therefore, it obtains accurate mobility measurements in bulk organic semiconductors.

However, when handling mobility as an inherent physical property, the type of organic semiconducting species (monomer, dimer, or multimer) depends on the purity, and may affect the TOF-SIMS measurement.

\section{Acknowledgement}

The author expresses appreciation for the provision of Compound (1) by Professor Chitoshi Kitamura in Shiga Prefectural University.

The author also expresses appreciation for the provision of Compound (2) by Professor Yutaka Takaguchi in Okayama University.

The author also expresses appreciation for the TOF photoconductivity measurements by Professor Tatsuo Arai and Professor Yoshinobu Nishimura at the University of Tsukuba.

TOF-SIMS and SIMS analysis were supported by Hitachi Urban Support Ltd. and Japan Display Inc.

The authors would like to thank Enago (www.enago.jp) for the English language review. 
1 Bendikov M, Wudl F, Perepichka DF. Tetrathiafulvalenes, oligoacenenes, and their buckminsterfullerene derivatives: The brick and mortar of organic electronics. Chemical Reviews. 2004;104(11):4891-946.

2 Rogers JA, Someya T, Huang Y. Materials and mechanics for stretchable electronics. Science. 2010;327(5973):1603-7.

3 Diao Y, Tee BC, Giri G, Xu J, Kim DH, Becerril HA, Stoltenberg RM, Lee TH, Xue G, Mannsfeld SC, Bao Z. Solution coating of large-area organic semiconductor thin films with aligned single-crystalline domains. Nature Materials. 2013;12(7):665-71

4 Kopylov YA, Trofimova TN, Stolovitskii YM, Nemchenko AM. Izvestiya Vysshikh Uchebnykh Zavedenii, Fizika, 1964;(5):28-33

5 Struijk CW, Sieval AB, Dakhorst JE, van Dijk M, Kimkes P, Koehorst RB, Donker H, Schaafsma TJ, Picken SJ, van de Craats AM, Warman JM. Liquid crystalline perylene diimides: architecture and charge carrier mobilities. Journal of the American Chemical Society. 2000;122(45):11057-66

6 Scher H, Montroll EW. Anomalous transit-time dispersion in amorphous solids. Physical Review B: Solid State. 1975;12(6):2455-77.

7 Funahashi M, Hanna J. Fast hole transport in a new calamitic liquid crystal of 2-(4' -heptyloxyphenyl)-6-dodecylthiobenzothiazole. Physical Review Letters, 1997;78(11):2184-7.

8 Tsumura A, Koezuka H, Ando TJ. Macromolecular electronic device: Field-effect transistor with a polythiophene thin film. Applied Physics Letters. 1986;49(18):1210-2. 9 Kitamura C, Abe Y, Ohara T, Yoneda A, Kawase T, Kobayashi T, Naito H, Komatsu T. Synthesis and crystallochromy of 1, 4, 7, 10 $\square$ tetraalkyltetracenes: Tuning of solid $\square$ state optical properties of tetracenes by alkyl side - chain length. Chemistry-A European Journal. 2010;16:890-8

10 Takaguchi Y, Kaneko K, Hotta K, Tsuboi S, Komatsu T. Pentacene compounds with high solvent solubility and high-mobility transistors therewith. JP2008094838A. Apr 24, 2008.

11 Hart H, Rajakumar P. 2'-Substituted meta-terphenyls as building blocks for cyclophanes with intra-annular functionality. Tetrahedron. 1995;51(5):1313-36.

12 Okamoto H, Arai T, Sakuragi H, Tokumaru K. Highly selective cis-trans photoisomerization of 1-pyrenylethylenes: relations between the modes of isomerization and potential energy surfaces. Bulletin of the Chemical Society of Japan. 1990;63(10):2881-90.

13 Siders P, Cave RJ, Marcus RA. A model for orientation effects in electron-transfer reactions. Journal of Chemical Physics. 1984;81(12):5613-24.

14 Kavarnos GJ, Turro NJ, Photosensitization by reversible electron transfer: theories, experimental evidence, and examples. Chemical Reviews. 1986;86(2):401-49.

15 Takahashi Y, Nakao H, Kumai R, Ishibashi S, Horiuchi S, Kohyama M, Kobayashi K, Yamasaki Y, Okamoto J, Sudayama T, Murakami Y, Tokura Y. Molecular electronic states in charge transfer complex studied by x-ray absorption spectroscopy. Journal of Physics: Conference Series. Conference on Light and Particle Beams in Materials Science. 2014;502(1):012036.

16 Schreiber F. Structure and growth of self-assembling monolayers. Progress in Surface Science. 2000;65(5-6):151-256.

17 Aikens CM. Electronic and geometric structure, optical properties, and excited state behavior in atomically precise thiolate-stabilized noble metal nanoclusters. Accounts of 
chemical research. 2018;51(12):3065-73.

18 Jakobsson M, Stafström S. Polaron effects and electric field dependence of the charge carrier mobility in conjugated polymers. Journal of Chemical Physics, 2011;135(13):134902/1-134902/9 